\title{
Pluricomplex Green's functions and Fano manifolds
}

\author{
Nicholas McCleerey and Valentino Tosatti
}

\begin{abstract}
We show that if a Fano manifold does not admit Kähler-Einstein metrics then the Kähler potentials along the continuity method subconverge to a function with analytic singularities along a subvariety which solves the homogeneous complex Monge-Ampère equation on its complement, confirming an expectation of Tian-Yau.
\end{abstract}

Keywords. Fano manifold; pluricomplex Green function; algebraic Kähler potentials

2010 Mathematics Subject Classification. 32W20 (primary); 32U35, 14J45, 32Q20, 53C25 (secondary)

\section{[Français]}

Titre. Fonctions de Green pluricomplexes et variétés de Fano

Résumé. Nous montrons que si une variété de Fano n'admet aucune métrique de Kähler-Einstein alors, suivant la méthode de continuité, les potentiels kählériens sous-convergent vers une fonction à singularités analytiques le long d'une sous-variété, sur le complémentaire de laquelle la fonction est solution de l'équation de Monge-Ampère complexe homogène. Cela confirme une attente de Tian-Yau.

Received by the Editors on June 28, 2018.

Accepted on May 30, 2019.

Nicholas McCleerey

Department of Mathematics, Northwestern University, 2033 Sheridan Road, Evanston, IL 60208, USA

e-mail:njm2@math.northwestern.edu

Valentino Tosatti

Department of Mathematics, Northwestern University, 2033 Sheridan Road, Evanston, IL 60208, USA

e-mail: tosatti@math.northwestern.edu

Partially supported by NSF RTG grant DMS-1502632 and by NSF grant DMS-1610278. 


\section{Contents}

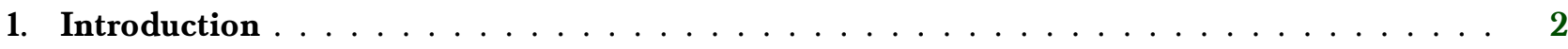

2. The partial $C^{0}$ estimate $\ldots \ldots \ldots \ldots \ldots \ldots \ldots \ldots \ldots$

3. The singularity model function $\ldots \ldots \ldots \ldots \ldots$

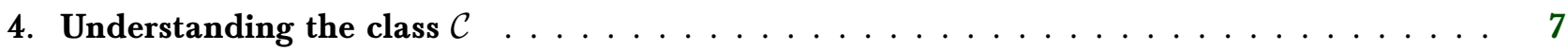

5. The pluricomplex Green's function $\ldots \ldots \ldots \ldots \ldots$

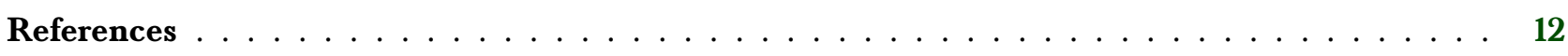

\section{Introduction}

Let $X^{n}$ be a Fano manifold, i.e. a compact complex manifold with $c_{1}(X)>0$. A Kähler-Einstein metric on $X$ is a Kähler metric $\omega$ which satisfies

$$
\operatorname{Ric}(\omega)=\omega .
$$

This implies that $[\omega]=c_{1}(X)$. We assume throughout this paper that $X$ does not admit a Kähler-Einstein metric. This is known to be equivalent to K-unstability by [13] (see also [40]), but we will not use this fact.

We fix a Kähler metric $\omega$ with $[\omega]=c_{1}(X)$, with Ricci potential $F$ defined by $\operatorname{Ric}(\omega)=\omega+\sqrt{-1} \partial \bar{\partial} F$ (normalized by $\int_{X}\left(e^{F}-1\right) \omega^{n}=0$ ). We consider Kähler metrics $\omega_{t}$ with $\left[\omega_{t}\right]=c_{1}(X)$ which satisfy

$$
\operatorname{Ric}\left(\omega_{t}\right)=t \omega_{t}+(1-t) \omega .
$$

We can write $\omega_{t}=\omega+\sqrt{-1} \partial \bar{\partial} \varphi_{t}$ and the functions $\varphi_{t}$ solve the complex Monge-Ampère equation [46]

$$
\omega_{t}^{n}=e^{F-t \varphi_{t}} \omega^{n}
$$

A solution $\varphi_{t}$ exists on $[0, R(X))$ where $R(X) \leqslant 1$ is the greatest lower bound for the Ricci curvature of Kähler metrics in $c_{1}(X)$ [36]. It is known [35, 38] that since $X$ does not admit Kähler-Einstein metrics, we must have that $\lim _{t \rightarrow R(X)} \sup _{X} \varphi_{t}=+\infty$. We fix a sequence $t_{i} \rightarrow R(X)$ and write $\varphi_{i}:=\varphi_{t_{i}}$ and $\omega_{i}:=\omega_{t_{i}}$. Using this result, together with multiplier ideal sheaves, Nadel [29, Proposition 4.1] proved that (up to passing to a subsequence) the measures $\omega_{i}^{n}$ converge to zero (as measures) on compact sets of $X \backslash V$ for some proper analytic subvariety $V \subset X$, and in [44] the second-named author improved this to uniform convergence.

By weak compactness of closed positive currents in a fixed cohomology class, up to subsequences we can extract a limit $\rho$ of $\varphi_{i}-\sup _{X} \varphi_{i}$ (which may depend on the subsequence), which is an unbounded $\omega$-psh function, and the convergence happens in the $L^{1}$ topology.

In their work [41, p.178], Tian-Yau expressed the expectation that $\rho$ should have logarithmic poles along a proper analytic subvariety $V \subset X$, and that it should satisfy $(\omega+\sqrt{-1} \partial \bar{\partial} \rho)^{n}=0$ on $X \backslash V$, so that $\rho$ could be thought of as a kind of pluricomplex Green's function (see also [38, p.238] and [39, p.109]).

In this note we confirm Tian-Yau's expectation:

Theorem 1.1. Let $X$ be a Fano manifold without a Kähler-Einstein metric, and let $\omega_{t}=\omega+\sqrt{-1} \partial \bar{\partial} \varphi_{t}$ be the solutions of the continuity method (1.1). Given any sequence $t_{i} \in[0, R(X))$ with $t_{i} \rightarrow R(X)$, choose a subsequence such that $\varphi_{t_{i}}-\sup _{X} \varphi_{t_{i}}$ converge in $L^{1}(X)$ to an $\omega$-psh function $\rho$. Then we can find $m \geqslant 1$ and an $\omega$-psh function $\psi$ on $X$ with analytic singularities

$$
\psi=\frac{1}{m} \log \sum_{j=1}^{p} \lambda_{j}^{2}\left|S_{j}\right|_{h^{m}}^{2},
$$


for some $\lambda_{j} \in(0,1]$ and some sections $S_{j} \in H^{0}\left(X, K_{X}^{-m}\right)$, with nonempty common zero locus $V \subset X$ such that $\rho-\psi$ is bounded on $X$, and on $X \backslash V$ we have

$$
(\omega+\sqrt{-1} \partial \bar{\partial} \rho)^{n}=0,
$$

where the Monge-Ampère product is in the sense of Bedford-Taylor [6].

In particular, Theorem 1.1 implies that the non-pluripolar Monge-Ampère operator of $\rho$ (defined in [12]) vanishes identically on $X$. On the other hand, there is another meaningful Monge-Ampère operator that can be applied to $\rho$. Indeed, the fact that $\rho-\psi \in L^{\infty}(X)$ implies that $\rho$ itself has analytic singularities. In [3] Andersson-Błocki-Wulcan defined a Monge-Ampère operator for $\omega$-psh functions with analytic singularities (generalizing earlier work of Andersson-Wulcan [4] in the local setting). In general, applying this MongeAmpère operator to $\rho$ will produce a Radon measure $\mu$ on $X$ (which may be identically zero in some cases), which by Theorem 1.1 is supported on the analytic set $V$, thus providing geometrically interesting examples of unbounded quasi-psh functions on compact Kähler manifolds with Monge-Ampère operator concentrated on a subvariety (see also [1, 2] for related results in the local setting). In particular, this answers [11, Question 1 (c)], an open problem raised at the AIM workshop "The complex Monge-Ampère equation" in August 2016 (cf. the related [23, Question 12]).

Note that in general a formula for the total mass of $\mu$ is proved in [3, Theorem 1.2], and it satisfies

$$
\int_{X} \mu \leqslant \int_{X} \omega^{n}
$$

with strict inequality in general (but it is not hard to see that if $\operatorname{dim} X=2$ and $V$ is a finite set then equality holds). Therefore, the measure $\mu$ is in general different from the measures that one obtains as weak limits of $\left(\omega+\sqrt{-1} \partial \bar{\partial} \varphi_{i}\right)^{n}$ (up to subsequences), whose total mass is always equal to $\int_{X} \omega^{n}$.

Remark 1.2. [Remark added in proof] After this work was posted on the arXiv, and partly prompted by it, Błocki [10] modified the definition of the Monge-Ampère operator for $\omega$-psh functions with analytic singularities of $[4,3]$, and with his definition the total mass is always equal to $\int_{X} \omega^{n}$. It is an interesting question to determine whether this Monge-Ampère operator equals the weak limit of $\left(\omega+\sqrt{-1} \partial \bar{\partial} \varphi_{i}\right)^{n}$.

Remark 1.3. As in the second-named author's previous work [44], Theorem 1.1 has a direct counterpart for solutions of the normalized Kähler-Ricci flow, instead of the continuity method (1.1). The statement is identical to Theorem 1.1, except that now the sequence $t_{i}$ goes to $+\infty$. The proof is also almost verbatim the same, and the partial $C^{0}$ estimate along the flow is proved in [14,15] (see also [5]). All other ingredients used also have well-known counterparts for the flow (see [44]). We leave the simple details to the interested reader.

Remark 1.4. The behavior of the solutions $\omega_{t}$ of $(1.1)$ as $t \rightarrow R(X)$ has been investigated in the past. If the manifold is K-stable, [20] show that $\omega_{t}$ converge smoothly to a Kähler-Einstein metric. If on the other hand no such metric exists, the blowup behavior of $\omega_{t}$ has been investigated in [29,44] in the setting of this paper, and also in $[20,26,33]$ by allowing reparametrizations of the metrics by diffeomorphisms.

The proof of Theorem 1.1 relies on the partial $C^{0}$ estimate for solutions of (1.1) which was established by Székelyhidi [37]. We recall this in section 2, together with a well-known reformulation of this estimate (Proposition 2.1). In section 3 we observe that this gives us the singularity model function $\psi$ in (1.2), and it also implies that $\rho$ has the same singularity type as $\psi$. In section 4 we show the general fact that every $\omega$-psh function on $X$ with the same singularity type as $\psi$ has vanishing Monge-Ampère operator outside $V$, thus proving Theorem 1.1. This relies on a geometric understanding of the rational map defined by the sections $\left\{S_{j}\right\}$ as in Theorem 1.1. Lastly, in section 5 we discuss the pluricomplex Green's function with the same singularity type as $\psi$. 
Acknowledgments. We thank Z. Błocki, T.C. Collins, S. Kolodziej and D.H. Phong for discussions on this topic at the AIM workshop "The complex Monge-Ampère equation" in August 2016, E. Wulcan for her interest in this work and A. Rashkovskii and the referee for useful comments. The second-named author is also grateful to S.-T. Yau for related discussions over the years. This work was completed during the second-named author's visit to the Institut Henri Poincaré in Paris (supported by a Chaire Poincaré funded by the Clay Mathematics Institute) which he would like to thank for the hospitality and support.

\section{The partial $C^{0}$ estimate}

To start we fix some notation. We choose a Hermitian metric $h$ on $K_{X}^{-1}$ with curvature $R_{h}=\omega$ (such $h$ is unique up to scaling), and let $h^{m}$ be the induced metric on $K_{X}^{-m}$, for all $m \geqslant 1$. Let $N_{m}=\operatorname{dim} H^{0}\left(X, K_{X}^{-m}\right)$, and for any $m \geqslant 1$ define the density of states function

$$
\rho_{m}(\omega)=\sum_{j=1}^{N_{m}}\left|S_{j}\right|_{h^{m}}^{2},
$$

where $S_{1}, \ldots, S_{N_{m}}$ are a basis of $H^{0}\left(X, K_{X}^{-m}\right)$ which is orthonormal with respect to the $L^{2}$ inner product $\int_{X}\left\langle S_{1}, S_{2}\right\rangle_{h^{m}} \omega^{n}$. Clearly $\rho_{m}(\omega)$ is independent of the choice of basis, and is also unchanged if we scale $h$ by a constant. The integral $\int_{X} \rho_{m}(\omega) \omega^{n}$ equals $N_{m}$, and if $m$ is sufficiently large so that $K_{X}^{-m}$ is very ample, then $\rho_{m}(\omega)$ is strictly positive on $X$. If we apply this same construction to the metrics $\omega_{t}$ and Hermitian metrics $h_{t}=h e^{-\varphi_{t}}$ we get a density of states function $\rho_{m}\left(\omega_{t}\right)$. Following [39], we say that a "partial $C^{0}$ estimate" holds if there exist $m \geqslant 1$ and a constant $C>0$ such that

$$
\inf _{X} \rho_{m}\left(\omega_{t}\right) \geqslant C^{-1},
$$

holds for all $t \in[0, R(X))$. The reason for this name is explained by the following proposition, which is essentially well-known (see [39, Lemma 2.2] and [43, Proposition 5.1]), but we provide the details for convenience:

Proposition 2.1. If a partial $C^{0}$ estimate holds then there exists $m \geqslant 1$, such that for all $\varepsilon>0$ we can find a constant $C>0$ so that for all $t \in[\varepsilon, R(X))$ we can find real numbers $1=\lambda_{1}(t) \geqslant \ldots \geqslant \lambda_{N_{m}}(t)>0$ and a basis $\left\{S_{j}(t)\right\}_{1 \leqslant j \leqslant N_{m}}$ of $H^{0}\left(X, K_{X}^{-m}\right)$, orthonormal with respect to the $L^{2}$ inner product of $\omega, h^{m}$, such that for all $t \in[0, R(X))$ we have

$$
\left.\sup _{X}\left|\varphi_{t}-\sup _{X} \varphi_{t}-\frac{1}{m} \log \sum_{j=1}^{N_{m}} \lambda_{j}(t)^{2}\right| S_{j}(t)\right|_{h^{m}} ^{2} \mid \leqslant C .
$$

In the rest of the paper we will fix a value of $\varepsilon>0$ once and for all, for example $\varepsilon=R(X) / 2$. The precise choice is irrelevant, since we are only interested in the behavior as $t \rightarrow R(X)$.

Proof. First, it is well-known that for all $m \geqslant 1$ and $\varepsilon>0$ there is a constant $C$ such that for all $t \in[\varepsilon, R(X))$ we have

$$
\rho_{m}\left(\omega_{t}\right) \leqslant C .
$$

To see this, first observe for every $S \in H^{0}\left(X, K_{X}^{-m}\right)$ we have

$$
\Delta_{\omega_{t}}|S|_{h_{t}^{m}}^{2}=|\nabla S|_{t}^{2}-2 m|S|_{h_{t}^{m}}^{2} \geqslant-2 m|S|_{h_{t}^{m}}^{2},
$$

and that since $\operatorname{Ric}\left(\omega_{t}\right) \geqslant t \omega_{t} \geqslant \varepsilon \omega_{t}$, Myers' Theorem gives a uniform upper bound for $\operatorname{diam}\left(X, \omega_{t}\right)$ and then Croke [19] and Li [27] show that the Sobolev constant of $\left(X, \omega_{t}\right)$ has a uniform upper bound. We can then apply Moser iteration to (2.4) to get

$$
\sup _{X}|S|_{h_{t}^{m}}^{2} \leqslant C \int_{X}|S|_{h_{t}^{m}}^{2} \omega_{t}^{n} \leqslant C,
$$


provided we assume that $\int_{X}|S|_{h_{t}^{m}}^{2} \omega_{t}^{n}=1$. Taking now an orthonormal basis of sections and summing we obtain (2.3).

Thanks to (2.3) we know that for $t \in[\varepsilon, R(X))$ a partial $C^{0}$ estimate is equivalent to

$$
\sup _{X}\left|\log \rho_{m}\left(\omega_{t}\right)\right| \leqslant C .
$$

We now take a basis $\left\{\tilde{S}_{j}(t)\right\}_{1 \leqslant j \leqslant N_{m}}$ of $H^{0}\left(X, K_{X}^{-m}\right)$ orthonormal with respect to the $L^{2}$ inner product of $\omega_{t}, h_{t}^{m}$ and notice that since $h_{t}^{m}=e^{-m \varphi_{t}} h^{m}$ we clearly have

$$
\varphi_{t}=\frac{1}{m} \log \frac{\sum_{j=1}^{N_{m}}\left|\tilde{S}_{j}(t)\right|_{h^{m}}^{2}}{\sum_{j=1}^{N_{m}}\left|\tilde{S}_{j}(t)\right|_{h_{t}^{m}}^{2}}
$$

which is equivalent to

$$
\varphi_{t}-\frac{1}{m} \log \sum_{j=1}^{N_{m}}\left|\tilde{S}_{j}(t)\right|_{h^{m}}^{2}=-\frac{1}{m} \log \rho_{m}\left(\omega_{t}\right) .
$$

It follows from (2.6) and (2.7) that that for $t \in[\varepsilon, R(X))$ a partial $C^{0}$ estimate is equivalent to an estimate

$$
\left.\sup _{X}\left|\varphi_{t}-\frac{1}{m} \log \sum_{j=1}^{N_{m}}\right| \tilde{S}_{j}(t)\right|_{h^{m}} ^{2} \mid \leqslant C .
$$

We now choose another basis $\left\{S_{j}\right\}_{1 \leqslant j \leqslant N_{m}}$ of $H^{0}\left(X, K_{X}^{-m}\right)$ orthonormal with respect to the $L^{2}$ inner product of $\omega, h^{m}$. After modifying $S_{j}$ and $\widetilde{S}_{j}(t)$ by $t$-dependent unitary transformations, we obtain orthonormal bases $\left\{S_{j}(t)\right\}_{1 \leqslant j \leqslant N_{m}}$ with respect to $\omega, h^{m}$, and $\left\{\tilde{S}_{j}(t)\right\}_{1 \leqslant j \leqslant N_{m}}$ with respect to $\omega_{t}, h_{t}^{m}$ such that

$$
\tilde{S}_{j}(t)=\mu_{j}(t) S_{j}(t)
$$

for some positive real numbers $\mu_{j}(t)$, with $\mu_{1}(t) \geqslant \ldots \geqslant \mu_{N_{m}}(t)>0$. We then let $\lambda_{j}(t)=\mu_{j}(t) / \mu_{1}(t)$ and we see that a partial $C^{0}$ estimate is equivalent to

$$
\left.\sup _{X}\left|\varphi_{t}-\frac{2}{m} \log \mu_{1}(t)-\frac{1}{m} \log \sum_{j=1}^{N_{m}} \lambda_{j}(t)^{2}\right| S_{j}(t)\right|_{h^{m}} ^{2} \mid \leqslant C .
$$

We now claim that if a partial $C^{0}$ estimate holds, then for all $t \in[\varepsilon, R(X))$ we also have

$$
\left|\frac{2}{m} \log \mu_{1}(t)-\sup _{X} \varphi_{t}\right| \leqslant C .
$$

Once this is proved, combining (2.8) and (2.9) we get (2.2). To prove (2.9), first use (2.5) to get

$$
C \geqslant \sup _{X}\left|\tilde{S}_{1}(t)\right|_{h_{t}^{m}}^{2} \geqslant \mu_{1}(t)^{2} \sup _{X}\left|S_{1}(t)\right|_{h^{m}}^{2} e^{-m \sup _{X} \varphi_{t}},
$$

and the fact $\int_{X}\left|S_{1}(t)\right|_{h^{m}}^{2} \omega^{n}=1$ implies that $\sup _{X}\left|S_{1}(t)\right|_{h^{m}}^{2} \geqslant 1 / \operatorname{Vol}(X, \omega)$, and so

$$
\left(\frac{2}{m} \log \mu_{1}(t)-\sup _{X} \varphi_{t}\right) \leqslant C \text {. }
$$

On the other hand the partial $C^{0}$ estimate (2.1) implies that

$$
C^{-1} \leqslant \rho_{m}\left(\omega_{t}\right)=\sum_{j=1}^{N_{m}}\left|\tilde{S}_{j}(t)\right|_{h_{t}^{m}}^{2} \leqslant \mu_{1}(t)^{2} \sum_{j=1}^{N_{m}}\left|S_{j}(t)\right|_{h^{m}}^{2} e^{-m \varphi_{t}},
$$


and we clearly have that

$$
\sup _{j} \sup _{X}\left|S_{j}(t)\right|_{h^{m}}^{2} \leqslant C,
$$

since the sections $\left\{S_{j}(t)\right\}$ are just varying in a compact unitary group (or one can also repeat the Moser iteration argument of (2.3) for the fixed metric $\omega$ ). This together with (2.10), evaluated at the point where $\varphi_{t}$ achieves its maximum, gives the reverse inequality

$$
\left(\sup _{X} \varphi_{t}-\frac{2}{m} \log \mu_{1}(t)\right) \leqslant C
$$

which completes the proof of (2.9).

\section{The singularity model function}

The next goal is to use the partial $C^{0}$ estimate in Proposition 2.1 to construct a singular $\omega$-psh function $\psi$ which will have the same singularity type of any weak limit of the normalized solutions $\varphi_{i}-\sup _{X} \varphi_{i}$ of the continuity method.

Let the notation be as in Proposition 2.1, and in particular we fix once and for all a value of $m \geqslant 1$ given there. We can find a sequence $t_{i} \rightarrow R(X)$ and an $\omega$-psh function $\rho$ with $\sup _{X} \rho=0$ such that $\varphi_{i}-\sup _{X} \varphi_{i} \rightarrow \rho$ in $L^{1}(X)$, and pointwise a.e. Passing to a subsequence, we can find a basis $\left\{S_{j}\right\}_{1 \leqslant j \leqslant N_{m}}$ of $H^{0}\left(X, K_{X}^{-m}\right)$ orthonormal with respect to the $L^{2}$ inner product of $\omega, h^{m}$, such that $S_{j}\left(t_{i}\right) \rightarrow S_{j}$ smoothly as $i \rightarrow \infty$, for all $1 \leqslant j \leqslant N_{m}$. The change of basis matrix from $\left\{S_{j}\right\}_{1 \leqslant j \leqslant N_{m}}$ to $\left\{S_{j}(t)\right\}_{1 \leqslant j \leqslant N_{m}}$ induces an automorphism $\sigma(t)$ of $\mathbb{C P}^{N_{m}-1}$, such that $\sigma\left(t_{i}\right) \rightarrow \operatorname{Id}$ smoothly as $i \rightarrow \infty$.

For ease of notation, write

$$
\psi_{t}=\frac{1}{m} \log \sum_{j=1}^{N_{m}} \lambda_{j}(t)^{2}\left|S_{j}(t)\right|_{h^{m}}^{2} .
$$

These functions are Kähler potentials for $\omega$ since

$$
\omega+\sqrt{-1} \partial \bar{\partial} \psi_{t}=\frac{l^{*} \sigma(t)^{*} \tau(t)^{*} \omega_{F S}}{m}>0
$$

where $\iota: X \hookrightarrow \mathbb{C P}^{N_{m}-1}$ is the Kodaira embedding map given by the sections $\left\{S_{j}\right\}_{1 \leqslant j \leqslant N_{m}}$, the map $\tau(t)$ is the automorphism of $\mathbb{C P}^{N_{m}-1}$ induced by the diagonal matrix with entries $\left\{\lambda_{j}(t)\right\}_{1 \leqslant j \leqslant N_{m}}$, and $\omega_{F S}$ is the Fubini-Study metric on $\mathbb{C P}^{N_{m}-1}$. The identity in (3.1) follows directly from the definition of the Fubini-Study metric $\omega_{F S}$ on $\mathbb{C P}^{N_{m}-1}$, which on $\mathbb{C}^{N_{m}} \backslash\{0\}$ is given explicitly by $\omega_{F S}=\sqrt{-1} \partial \bar{\partial} \log \sum_{j=1}^{N_{m}}\left|z_{j}\right|^{2}$, and from the fact that the curvature of $h$ is $\omega$.

Up to passing to a subsequence of $t_{i}$, we may assume that $\lambda_{j}\left(t_{i}\right) \rightarrow \lambda_{j}$ as $i \rightarrow \infty$ for all $j$, and we have

$$
1=\lambda_{1} \geqslant \ldots \geqslant \lambda_{p}>0=\lambda_{p+1}=\cdots=\lambda_{N_{m}},
$$

for some $1 \leqslant p<N_{m}$. The case $p=N_{m}$ is impossible because by (2.2) it would imply a uniform $L^{\infty}$ bound for $\varphi_{t}$ and so $X$ would admit a Kähler-Einstein metric. For the same reason, the set $V:=\left\{S_{1}=\cdots=S_{p}=0\right\}$ must be a nonempty proper analytic subvariety of $X$.

Note that thanks to $(2.2)$ we can write

$$
\omega_{t}^{n}=e^{F-t\left(\varphi_{t}-\sup _{X} \varphi_{t}\right)} e^{-t \sup _{X} \varphi_{t}} \omega^{n} \leqslant C e^{t \psi_{t}} e^{-t \sup _{X} \varphi_{t}} \omega^{n},
$$

and since the term $e^{t \psi_{t}}$ is uniformly bounded on compact sets of $X \backslash V$, we see immediately that

$$
\omega_{t}^{n} \rightarrow 0,
$$


uniformly on compact sets of $X \backslash V$ (this result was proved in [44] without using the partial $C^{0}$ estimate, which was not available at the time, with weaker results established earlier in [29]).

Let then

$$
\psi=\frac{1}{m} \log \sum_{j=1}^{p} \lambda_{j}^{2}\left|S_{j}\right|_{h^{m}}^{2},
$$

which is a smooth function on $X \backslash V$ which approaches $-\infty$ uniformly on $V$. Since $e^{m \psi_{t}} \rightarrow e^{m \psi}$ smoothly on $X$, and since $\psi_{t}$ are smooth and $\omega$-psh, it follows that $\psi$ is $\omega$-psh. This will be our singularity model function in the rest of the argument, as we now explain:

Lemma 3.1. Define the class

$$
\mathcal{C}=\left\{\eta \in P S H(X, \omega) \mid \eta-\psi \in L^{\infty}(X)\right\},
$$

of $\omega$-psh functions with the same singularity type as $\psi$. Then we have that $\rho \in \mathcal{C}$.

Proof. Recall that we have $\varphi_{i}-\sup _{X} \varphi_{i} \rightarrow \rho$ a.e. on $X$. Thanks to (2.2), the function $\rho$ satisfies

$$
|\rho-\psi| \leqslant C
$$

a.e. on $X$, which implies the same inequality on all of $X$ by elementary properties of psh functions (cf. [25, Theorem K.15]), thus showing that $\rho \in \mathcal{C}$.

\section{Understanding the class $\mathcal{C}$}

We now exploit the geometry of our setting to gain a better understanding of the class of functions $\mathcal{C}$.

The sections $\left\{\lambda_{j} S_{j}\right\}_{1 \leqslant j \leqslant p}$ define a rational map $\Phi: X \rightarrow \mathbb{C P}^{p-1}$, with indeterminacy locus $Z \subset V$ (this inclusion is in general strict, since $\operatorname{codim} Z \geqslant 2$ while $V$ may contain divisorial components). Let $Y$ be the image of $\Phi$, i.e. the closure of $\Phi(X \backslash Z)$ in $\mathbb{C P}^{p-1}$, which is an irreducible projective variety. By resolving the indeterminacies of $\Phi$ we get a modification $\mu: \tilde{X} \rightarrow X$, obtained as a sequence of blowups with smooth centers, and a holomorphic map $\Psi: \tilde{X} \rightarrow Y$ such that $\Psi=\Phi \circ \mu$ holds on $\tilde{X} \backslash \mu^{-1}(Z)$. We may also assume without loss of generality that $\mu$ principalizes the ideal sheaf generated by $\left\{S_{j}\right\}_{1 \leqslant j \leqslant p}$, so that we have

$$
\mu^{*}(\omega+\sqrt{-1} \partial \bar{\partial} \psi)=\theta+[E],
$$

where $E$ is an effective $\mathbb{R}$-divisor with $\mu(E) \subset V$, and $\theta$ is a smooth closed semipositive $(1,1)$ form on $\tilde{X}$. We will denote by $\omega_{F S, p}$ the Fubini-Study metric on $\mathbb{C P}^{p-1}$. To identify $\theta$, note that on $X \backslash V$ we have by definition $\omega+\sqrt{-1} \partial \bar{\partial} \psi=\frac{\Phi^{*} \omega_{F S, p}}{m}$, and so on $\tilde{X} \backslash \mu^{-1}(V)$ we have

$$
\mu^{*}(\omega+\sqrt{-1} \partial \bar{\partial} \psi)=\frac{\mu^{*} \Phi^{*} \omega_{F S, p}}{m}=\frac{\Psi^{*} \omega_{F S, p}}{m},
$$

and so $\theta=\frac{\Psi^{*} \omega_{F S, p}}{m}$ on $\tilde{X} \backslash \mu^{-1}(V)$, and hence everywhere since both sides of this equality are smooth forms on all of $\tilde{X}$. This proves the key relation

$$
\mu^{*}(\omega+\sqrt{-1} \partial \bar{\partial} \psi)=\frac{\Psi^{*} \omega_{F S, p}}{m}+[E]
$$

Let $\tilde{X} \stackrel{v}{\rightarrow} \tilde{Y} \stackrel{q}{\rightarrow} Y$ be the Stein factorization of $\Psi$, where $\tilde{Y}$ is an irreducible projective variety, the map $v$ has connected fibers, and $q$ is a finite morphism.

We have that $q^{*} \omega_{F S, p}$ is a smooth semipositive $(1,1)$ form on $\tilde{Y}$, in the sense of analytic spaces. Since $v$ has compact connected fibers, a standard argument shows that the set of $\frac{\Psi^{*} \omega_{F S, p}}{m}$-psh functions on $\tilde{X}$ 
can be identified with the set of (weakly) $\frac{q^{*} \omega_{F S, p}}{m}$-psh functions on $\tilde{Y}$ via $v^{*}$ (indeed the restriction of every $\frac{\Psi^{*} \omega_{F S, p}}{m}$-psh function to any fiber of $v$ is plurisubharmonic and hence constant on that fiber). We will use this standard argument several other times in the following.

Here and in the following, as in [21], a weakly quasi-psh function on a compact analytic space means a quasi-psh function on its regular part which is locally bounded above near the singular set. As shown in [21, §1], weakly quasi-psh functions are the same as usual quasi-psh functions if the analytic space is normal, and otherwise they can be identified with quasi-psh functions on its normalization.

Proposition 4.1. Given any function $\eta \in \mathcal{C}$, there is a unique bounded weakly $\frac{q^{*} \omega_{F S, p}}{m}$-psh function $u$ on $\tilde{Y}$ such that

$$
\mu^{*} \eta=\mu^{*} \psi+v^{*} u
$$

Conversely, given any bounded weakly $\frac{q^{*} \omega_{F S, p}}{m}$-psh function $u$ on $\tilde{Y}$ there is a unique function $\eta \in \mathcal{C}$ such that (4.2) holds.

The relation in (4.2) thus allows us to identify the class $\mathcal{C}$ with the class of bounded weakly $\frac{q^{*} \omega_{F S, p}}{m}$-psh functions on $\tilde{Y}$.

Next, we observe that

\section{Proposition 4.2. We have that}

$$
\operatorname{dim} Y<\operatorname{dim} X .
$$

This is a consequence of our assumption that $X$ does not admit a Kähler-Einstein metric.

Lastly, every function $\eta \in \mathcal{C}$ belongs to $L_{\text {loc }}^{\infty}(X \backslash V)$, and so its Monge-Ampère operator $(\omega+\sqrt{-1} \partial \bar{\partial} \eta)^{n}$ is well-defined on $X \backslash V$ thanks to Bedford-Taylor [6]. Combining the results in Propositions 4.1 and 4.2 we will obtain:

Theorem 4.3. For every $\eta \in \mathcal{C}$ we have that

$$
(\omega+\sqrt{-1} \partial \bar{\partial} \eta)^{n}=0
$$

on $X \backslash V$.

In particular, this holds for the function $\rho$, thanks to Lemma 3.1, and Theorem 1.1 thus follows from these.

Proof of Proposition 4.1. If $\eta$ is an $\omega$-psh function on $X$ with $\eta-\psi \in L^{\infty}(X)$, i.e. $\eta$ is an element of $\mathcal{C}$, then using (4.1) we can write

$$
\mu^{*}(\omega+\sqrt{-1} \partial \bar{\partial} \eta)=\frac{\Psi^{*} \omega_{F S, p}}{m}+\sqrt{-1} \partial \bar{\partial} \mu^{*}(\eta-\psi)+[E],
$$

where $E$ is as in $(4.1)$ and $\mu^{*}(\eta-\psi) \in L^{\infty}(\tilde{X})$. Applying the Siu decomposition, we see that

$$
\frac{\Psi^{*} \omega_{F S, p}}{m}+\sqrt{-1} \partial \bar{\partial} \mu^{*}(\eta-\psi) \geqslant 0,
$$

weakly, and so

$$
\mu^{*}(\eta-\psi)=v^{*} u_{\eta}
$$

for a bounded weakly $\frac{q^{*} \omega_{F S, p}}{m}$-psh functions $u_{\eta}$ on $\tilde{Y}$, which is uniquely determined by $\eta$ (and $\psi$, which we view as fixed). 
Conversely, given a bounded weakly $\frac{q^{*} \omega_{F S, p}}{m}$-psh function $u$ on $\tilde{Y}$, we have that $v^{*} u$ is $\frac{\Psi^{*} \omega_{F S, p}}{m}$-psh and bounded on $\tilde{X}$ and so

$$
0 \leqslant \frac{\Psi^{*} \omega_{F S, p}}{m}+[E]+\sqrt{-1} \partial \bar{\partial} v^{*} u=\mu^{*} \omega+\sqrt{-1} \partial \bar{\partial}\left(\mu^{*} \psi+v^{*} u\right),
$$

and so $\mu^{*} \psi+v^{*} u$ descends to an $\omega$-psh function $\eta_{u}$ on $X$ with $\eta_{u}-\psi \in L^{\infty}(X)$, i.e. $\eta_{u} \in \mathcal{C}$.

These two constructions are inverses to each other, and so we obtain the desired bijective correspondence between functions in $\mathcal{C}$ and bounded weakly $\frac{q^{*} \omega_{F S, p}}{m}$-psh functions on $\tilde{Y}$.

Proof of Proposition 4.2. On $X$ we have the estimate

$$
\omega_{t} \geqslant C^{-1} \frac{l^{*} \sigma(t)^{*} \tau(t)^{*} \omega_{F S}}{m},
$$

which is a direct consequence of the partial $C^{0}$ estimate (see e.g. [24, Lemma 4.2]). We can also give a direct proof by calculating

$$
\Delta_{\omega_{t}}\left(\log \operatorname{tr}_{\omega_{t}}\left(\frac{l^{*} \sigma(t)^{*} \tau(t)^{*} \omega_{F S}}{m}\right)-A\left(\varphi_{t}-\sup _{X} \varphi_{t}-\psi_{t}\right)\right) \geqslant \operatorname{tr}_{\omega_{t}}\left(\frac{l^{*} \sigma(t)^{*} \tau(t)^{*} \omega_{F S}}{m}\right)-C,
$$

if $A$ is sufficiently large, and applying the maximum principle together with the partial $C^{0}$ estimate (2.2) (for this calculation we used that the bisectional curvature of the metrics $\frac{t^{*} \sigma(t)^{*} \tau(t)^{*} \omega_{F S}}{m}$ have a uniform upper bound independent of $t$ ).

If we had $\operatorname{dim} Y=\operatorname{dim} X$ then the rational map $\Phi$ would be generically finite, so there would be a nonempty open subset $U \Subset X \backslash V$ such that $\left.\Phi\right|_{U}$ is a biholomorphism with its image. Recall that $\Phi$ is the rational map defined by the sections $\left\{\lambda_{j} S_{j}\right\}_{1 \leqslant j \leqslant p}$, while $\iota: X \hookrightarrow \mathbb{C P}^{N_{m}-1}$ is the embedding defined by the sections $\left\{S_{j}\right\}_{1 \leqslant j \leqslant N_{m}}$, and so $\Phi=\tilde{\tau} \circ P \circ \iota$ where $P: \mathbb{C P}^{N_{m}-1} \rightarrow \mathbb{C P}^{p-1}$ is the linear projection given by $\left[z_{1}: \cdots: z_{N_{m}}\right] \mapsto\left[z_{1}: \cdots: z_{p}\right]$ and $\tilde{\tau}: \mathbb{C P}^{p-1} \rightarrow \mathbb{C P}^{p-1}$ is the automorphism given by

$$
\left[z_{1}: \cdots: z_{p}\right] \mapsto\left[\lambda_{1} z_{1}: \cdots: \lambda_{p} z_{p}\right] .
$$

In particular, on the embedded open $n$-fold $\iota(U)$, we have that $\left.P\right|_{\iota(U)}$ is also a biholomorphism with its image. The automorphisms $\tau\left(t_{i}\right)$ descend to automorphisms $\tilde{\tau}\left(t_{i}\right)$ on $\mathbb{C P}^{p-1}$, and now as $i \rightarrow \infty$ these converge smoothly to the automorphism $\tilde{\tau}$. Thus $P \circ \tau\left(t_{i}\right) \circ \sigma\left(t_{i}\right) \circ \iota=\tilde{\tau}\left(t_{i}\right) \circ P \circ \sigma\left(t_{i}\right) \circ l$, which converge smoothly as maps to $\tilde{\tau} \circ P \circ \iota=\Phi$ on $U$ as $i \rightarrow \infty$.

Since $\Phi$ is an isomorphism on $U$, smooth convergence gives us that $P \circ \tau\left(t_{i}\right) \circ \sigma\left(t_{i}\right) \circ \iota$ is a local isomorphism. Thus, after possibly shrinking $U$,

$$
P:\left(\tau\left(t_{i}\right) \circ \sigma\left(t_{i}\right) \circ \iota\right)(U) \rightarrow\left(P \circ \tau\left(t_{i}\right) \circ \sigma\left(t_{i}\right) \circ \iota\right)(U)=\left(\tilde{\tau}\left(t_{i}\right) \circ P \circ \sigma\left(t_{i}\right) \circ \iota\right)(U)
$$

is an isomorphism, and for $i$ large the open sets $\left(\tilde{\tau}\left(t_{i}\right) \circ P \circ \sigma\left(t_{i}\right) \circ \iota\right)(U) \subset \mathbb{C P}^{p-1}$ converge to the open set $(\tilde{\tau} \circ P \circ \iota)(U)$ in the Hausdorff sense. Up to shrinking $U$, there is an open subset $V \subset \mathbb{C P}^{p-1}$ that contains $\left(\tilde{\tau}\left(t_{i}\right) \circ P \circ \sigma\left(t_{i}\right) \circ \iota\right)(U)$ for all $i$ large, and still $P^{-1}$ is well-defined on $V$ (and $P: P^{-1}(V) \rightarrow V$ is a biholomorphism), so that $P^{-1}(V)$ contains $\left(\tau\left(t_{i}\right) \circ \sigma\left(t_{i}\right) \circ \iota\right)(U)$ for all $i$ large, and on $P^{-1}(V)$ we have

$$
P^{*} \omega_{F S, p} \leqslant C \omega_{F S}
$$

On $U$ we also have that $\frac{\iota^{*} \sigma\left(t_{i}\right)^{*} \tau\left(t_{i}\right)^{*} P^{*} \omega_{F S, p}}{m}$ converges smoothly to $\frac{\Phi^{*} \omega_{F S, p}}{m}$, which is a Kähler metric on $U$. Thanks to (4.3) and (4.4), on $U$ we have

$$
\omega_{i} \geqslant C^{-1} \frac{l^{*} \sigma\left(t_{i}\right)^{*} \tau\left(t_{i}\right)^{*} \omega_{F S}}{m} \geqslant C^{-1} \frac{l^{*} \sigma\left(t_{i}\right)^{*} \tau\left(t_{i}\right)^{*} P^{*} \omega_{F S, p}}{m} \geqslant C^{-1} \frac{\Phi^{*} \omega_{F S, p}}{m},
$$

for all $i$ large, which implies that $\int_{U} \omega_{i}^{n} \geqslant C^{-1}$, which is absurd thanks to (3.2). 
Remark 4.4. In particular we see that if $\operatorname{dim} Y=0$ (i.e. $Y$ is a point) then we have $\mathcal{C}=\{\psi+s\}_{s \in \mathbb{R}}$. On the other hand as long as $\operatorname{dim} Y>0$ the class $\mathcal{C}$ is always rather large.

Proof of Theorem 4.3. Thanks to Proposition 4.1, every $\eta \in \mathcal{C}$ satisfies $\mu^{*} \eta=\mu^{*} \psi+v^{*} u$ for some bounded weakly $\frac{q^{*} \omega_{F S, p}}{m}$-psh function $u$ on $\tilde{Y}$. Then using (4.1) we have

$$
\begin{aligned}
\mu^{*}(\omega+\sqrt{-1} \partial \bar{\partial} \eta) & =\frac{\Psi^{*} \omega_{F S, p}}{m}+\sqrt{-1} \partial \bar{\partial} v^{*} u+[E] \\
& =v^{*}\left(\frac{q^{*} \omega_{F S, p}}{m}+\sqrt{-1} \partial \bar{\partial} u\right)+[E],
\end{aligned}
$$

and so if $K$ is any compact subset of $X \backslash V$, since $\mu$ is an isomorphism on $\mu^{-1}(K)$, we get

$$
\begin{aligned}
\int_{K}(\omega+\sqrt{-1} \partial \bar{\partial} \eta)^{n} & =\int_{\mu^{-1}(K)} \mu^{*}(\omega+\sqrt{-1} \partial \bar{\partial} \eta)^{n} \\
& =\int_{\mu^{-1}(K)} v^{*}\left(\frac{q^{*} \omega_{F S, p}}{m}+\sqrt{-1} \partial \bar{\partial} u\right)^{n}=0,
\end{aligned}
$$

since $\operatorname{dim} \tilde{Y}=\operatorname{dim} Y<\operatorname{dim} X$ by Proposition 4.2.

\section{The pluricomplex Green's function}

We can also consider the pluricomplex Green's function with singularity type determined by $\psi$, namely

$$
G=\sup \{u \mid u \in P S H(X, \omega), u \leqslant 0, u \leqslant \psi+O(1)\}^{*},
$$

which is the compact manifold analog of the construction in [31], and has been studied in detail in [18, 30,32] and references therein. In particular, since $\psi$ has analytic singularities, it follows from [31,32] that $G \in \mathcal{C}$.

Thanks to Proposition 4.1 we can write

$$
\mu^{*} G=\mu^{*} \psi+v^{*} F
$$

for a bounded weakly $\frac{q^{*} \omega_{F S, p}}{m}$-psh function $F$ on $\tilde{Y}$. The function $F$ is itself given by a suitable envelope.

Proposition 5.1. The pluricomplex Green's function $G$ satisfies (5.2) where $F$ is the envelope on $\tilde{Y}$ given by

$$
F=\sup \left\{w \mid w \in \operatorname{PSH}\left(\tilde{Y}, q^{*} \omega_{F S, p} / m\right), w \leqslant-v_{*} \mu^{*} \psi\right\}^{*},
$$

and where we are writing

$$
v_{*}(f)(y)=\sup _{x \in \mathcal{v}^{-1}(y)} f(x)
$$

for any function $f$ on $\tilde{X}, y \in \tilde{Y}$.

In other words, $F$ is given by a quasi-psh envelope with obstacle $-v_{*} \mu^{*} \psi$ on $\tilde{Y}$.

Proof. Write $E=\sum_{i} \lambda_{i} E_{i}$ for $E_{i}$ prime divisors and $\lambda_{i} \in \mathbb{R}_{>0}$, and for each $i$ fix a defining section $s_{i}$ of $\mathcal{O}\left(E_{i}\right)$ and a smooth metric $h_{i}$ on $\mathcal{O}\left(E_{i}\right)$ with curvature form $R_{i}$. For brevity, we will write $|s|_{h}^{2}=\prod_{i}\left|s_{i}\right|_{h_{i}}^{2 \lambda_{i}}$ and $R_{h}=\sum_{i} \lambda_{i} R_{i}$. Then the Poincaré-Lelong formula gives

$$
[E]=\sqrt{-1} \partial \bar{\partial} \log |s|_{h}^{2}+R_{h}
$$


and we obtain that $\mu^{*} \omega-R_{h}$ is cohomologous to $\frac{\Psi^{*} \omega_{F S, p}}{m}$ and

$$
\mu^{*} \omega-R_{h}=\frac{\Psi^{*} \omega_{F S, p}}{m}+\sqrt{-1} \partial \bar{\partial}\left(\log |s|_{h}^{2}-\mu^{*} \psi\right),
$$

and $\mu^{*} \psi-\log |s|_{h}^{2}$ is smooth on all of $\tilde{X}$. Note that if we denote by

$$
\tilde{G}=\sup \left\{\left.u\left|u \in P S H\left(\tilde{X}, \mu^{*} \omega\right), u \leqslant 0, u \leqslant \log \right| s\right|_{h} ^{2}+O(1)\right\}^{*},
$$

then we have that $\tilde{G}=\mu^{*} G$ (this is again because every $\mu^{*} \omega$-psh function on $\tilde{X}$ is in fact the pullback of an $\omega$-psh function on $X)$.

As in [28], we use a trick from [8, Section 4] (see also [31]), to show that

$$
\tilde{G}=\log |s|_{h}^{2}+\sup \left\{\left.v\left|v \in P S H\left(\tilde{X}, \mu^{*} \omega-R_{h}\right), v \leqslant-\log \right| s\right|_{h} ^{2}\right\}^{*} .
$$

For the reader's convenience, we supply the simple proof. Denote the right hand side by $\hat{G}$. For one direction, if $v$ is $\left(\mu^{*} \omega-R_{h}\right)$-psh and satisfies $v \leqslant-\log |s|_{h}^{2}$, then $u:=v+\log |s|_{h}^{2}$ satisfies $u \leqslant 0$ but also since $v \leqslant C$ on $\tilde{X}$, we see that $u \leqslant \log |s|_{h}^{2}+C$, and also

$$
\begin{aligned}
\mu^{*} \omega+\sqrt{-1} \partial \bar{\partial} u & =\mu^{*} \omega+\sqrt{-1} \partial \bar{\partial} \log |s|_{h}^{2}+\sqrt{-1} \partial \bar{\partial} v \\
& =\mu^{*} \omega-R_{h}+[E]+\sqrt{-1} \partial \bar{\partial} v \\
& \geqslant \mu^{*} \omega-R_{h}+\sqrt{-1} \partial \bar{\partial} v \geqslant 0,
\end{aligned}
$$

and so $\hat{G} \leqslant \tilde{G}$. Conversely, if $u$ is $\mu^{*} \omega$-psh and satisfies $u \leqslant 0$ and $u \leqslant \log |s|_{h}^{2}+C$ for some $C$, then the Siu decomposition of $\mu^{*} \omega+\sqrt{-1} \partial \bar{\partial} u$ contains $[E]$ and so

$$
0 \leqslant \mu^{*} \omega+\sqrt{-1} \partial \bar{\partial} u-[E]=\mu^{*} \omega-R_{h}+\sqrt{-1} \partial \bar{\partial}\left(u-\log |s|_{h}^{2}\right),
$$

and so $v:=u-\log |s|_{h}^{2}$ is $\left(\mu^{*} \omega-R_{h}\right)$-psh and satisfies $v \leqslant-\log |s|_{h}^{2}$, and it follows that $\tilde{G} \leqslant \hat{G}$, which proves our claim.

But finally note that for all $x \in \tilde{X}$ we have

$$
\begin{aligned}
& \log |s|_{h}^{2}(x)+\sup \left\{\left.v(x)\left|v \in \operatorname{PSH}\left(\tilde{X}, \mu^{*} \omega-R_{h}\right), v \leqslant-\log \right| s\right|_{h} ^{2}\right\} \\
& =\mu^{*} \psi(x)+\sup \left\{v(x) \mid v \in \operatorname{PSH}\left(\tilde{X}, \Psi^{*} \omega_{F S, p} / m\right), v \leqslant-\mu^{*} \psi\right\} \\
& =\mu^{*} \psi(x)+\sup \left\{w(v(x)) \mid w \in \operatorname{PSH}\left(\tilde{Y}, q^{*} \omega_{F S, p} / m\right), w \leqslant-v_{*} \mu^{*} \psi\right\}
\end{aligned}
$$

and taking the upper-semicontinuous regularization and using the claim above gives $\mu^{*} G=\mu^{*} \psi+\nu^{*} F$, which completes the proof.

Using Proposition 5.1 we can see that $F$ is continuous on a Zariski open subset of $\tilde{Y}$, using the following argument. Let $g: Y^{\prime} \rightarrow \tilde{Y}$ be a resolution of the singularities of $\tilde{Y}$. Then we have:

$$
g^{*} F=\sup \left\{w \mid w \in P S H\left(Y^{\prime}, g^{*} q^{*} \omega_{F S, p} / m\right), w \leqslant-g^{*} v_{*} \mu^{*} \psi\right\}^{*} .
$$

Note that $g^{*} q^{*} \omega_{F S, p} / m$ is semi-positive and big, and that $-g^{*} v_{*} \mu^{*} \psi$ is continuous off of $g^{-1}\left(v\left(\mu^{-1}\left(\psi^{-1}(-\infty)\right)\right)\right)$, where it is unbounded. Using the trick in [28], we can replace the obstacle $-g^{*} \mathcal{V}_{*} \mu^{*} \psi$ with a globally continuous obstacle $h$ without changing $g^{*} F$. Now, approximate $h$ uniformly by smooth functions $h_{j}$. It is easy to see that the envelopes:

$$
F_{j}:=\sup \left\{w \mid w \in P S H\left(Y^{\prime}, g^{*} q^{*} \omega_{F S, p} / m\right), w \leqslant h_{j}\right\}^{*} .
$$

converge uniformly to $g^{*} F$. But then by [8], the $F_{j}$ are continuous away from the non-Kähler locus of $g^{*} q^{*} \omega_{F S, p} / m$ (a proper Zariski closed subset, see e.g. [12]), so we are done. 
Remark 5.2. One is naturally led to wonder about what the optimal regularity of $G$ is. The sharp $C^{1,1}$ regularity (on a Zariski open subset) of envelopes of the form (5.3) has been recently obtained in [17, 45] in Kähler classes and in [16] in nef and big classes (see also [7, 8, 9]) when the obstacle is smooth (or at least $C^{1,1}$ ), but in our case the regularity of $-v_{*} \mu^{*} \psi$ does not seem to be very good, especially near the points where $v$ is not a submersion.

On the other hand, the first-named author [28] has very recently obtained $C^{1,1}$ regularity (on a Zariski open subset) of envelopes with prescribed analytic singularities, which include those of the form (5.1), generalizing results in [32] in the case of line bundles. In our situation, the results of $[28,32]$ do not apply since in (5.1) the functions $u$ and $\psi$ are both $\omega$-psh (while for these results one would need them to be quasi-psh with respect to two different $(1,1)$-forms such that the cohomology class of their difference is big). Moreover, the main result of [28] also allows for $u$ and $\psi$ being both $\omega$-psh, but then needs the condition that the total mass of the non-pluripolar Monge-Ampère operator of $\psi$ be strictly positive. This is obviously not the case in our situation however, by Theorem 4.3 .

Remark 5.3. One possibly interesting approach to studying higher regularity of functions $v \in \mathcal{C}$ which are already continuous on $X \backslash V$ is the following. Suppose $\sup _{X} v=0$. Fix an $M>0$ and let $\Omega$ be the open set $\Omega:=\{v<-M\}$. Then one can easily show using the comparison principle and Theorem 4.3 that we have:

$$
\max \{v,-M\}=V_{\Omega}-M,
$$

where here $V_{\Omega}$ is the global (Siciak) extremal function for $\Omega$. In particular, one sees that $\Omega$ is regular. There is then a well-developed theory about Hölder continuous regularity for such functions (the so called HCP property), see e.g. [34]. It may be possible to use this theory to study $G$, if one can first show that it is continuous in at least a neighborhood of $V$. Another possibility may be to study regularity of the boundary of $\Omega$ - see the very end of [28].

Remark 5.4. On can also naturally ask whether the function $\rho$ (and therefore also its singularity type $\psi$ ) in Theorem 1.1 is actually independent of the choice of subsequence $t_{i}$, and also how regular $\rho$ is on $X \backslash V$. Our guess is that $\rho$ is indeed uniquely determined, and is smooth on $X \backslash V$. These properties would both follow if one could show that the map $\Phi: X \rightarrow Y$ is independent of the chosen subsequence, and that the corresponding function $u$ on $\tilde{Y}$ given by Lemma 3.1 and Proposition 4.1 which satisfies

$$
\mu^{*} \rho=\mu^{*} \psi+v^{*} u
$$

actually solves a suitable complex Monge-Ampère equation on $\tilde{Y}$. In a related setting of Calabi-Yau manifolds fibered over lower-dimensional spaces, such a limiting equation after collapsing the fibers was obtained by the second-named author in [42, Theorem 4.1].

Remark 5.5. Lastly, we can also ask whether the limit $\rho$ (if it is unique) is necessarily equal to the pluricomplex Green's function $G$ up to addition of a constant. By remark 4.4 this is the case if the rational map $\Phi$ is constant, so that $Y$ is a point. In general though this seems rather likely false.

\section{References}

[1] P. Åhag, U. Cegrell, R. Czyż, and H. H. Pham, Monge-Ampère measures on pluripolar sets, J. Math. Pures Appl. (9) 92 (2009), no. 6, 613-627. MR-2565845

[2] P. Åhag, U. Cegrell, and H. H. Pham, Monge-Ampère measures on subvarieties, J. Math. Anal. Appl. 423 (2015), no. 1, 94-105. MR-3273169

[3] M. Andersson, Z. Błocki, and E. Wulcan, On a Monge-Ampère operator for plurisubharmonic functions with analytic singularities, Indiana Univ. Math. J., to appear. 
[4] M. Andersson and E. Wulcan, Green functions, Segre numbers, and King's formula, Ann. Inst. Fourier (Grenoble) 64 (2014), no. 6, 2639-2657. MR-3331176

[5] R. H. Bamler, Convergence of Ricci flows with bounded scalar curvature, Ann. of Math. (2) 188 (2018), no. 3, 753-831. MR-3866886

[6] E. Bedford and B. A. Taylor, The Dirichlet problem for a complex Monge-Ampère equation, Invent. Math. 37 (1976), no. 1, 1-44. MR-0445006

[7] R. J. Berman, Bergman kernels and equilibrium measures for line bundles over projective manifolds, Amer. J. Math. 131 (2009), no. 5, 1485-1524. MR-2559862

[8] R. J. Berman, From Monge-Ampère equations to envelopes and geodesic rays in the zero temperature limit, Math. Z. 291 (2019), no. 1-2, 365-394. MR-3936074

[9] R. J. Berman and J.-P. Demailly, Regularity of plurisubharmonic upper envelopes in big cohomology classes. In: Perspectives in analysis, geometry, and topology, pp. 39-66, Progr. Math., vol. 296, Birkhäuser/Springer, New York, 2012. MR-2884031

[10] Z. Błocki, On the complex Monge-Ampère operator for quasi-plurisubharmonic functions with analytic singularities, Bull. Lond. Math. Soc., to appear.

[11] Z. Błocki, M. Păun, and V. Tosatti (organizers), The complex Monge-Ampère equation, American Institute of Mathematics Workshop, Palo Alto, California, August 15-19, 2016. Report and open problems available at http://aimath.org/pastworkshops/mongeampere.html

[12] S. Boucksom, P. Eyssidieux, V. Guedj, and A. Zeriahi, Monge-Ampère equations in big cohomology classes, Acta Math. 205 (2010), no. 2, 199-262. MR-2746347

[13] X. Chen. S. K. Donaldson, and S. Sun, Kähler-Einstein metrics on Fano manifolds. I, II, III, J. Amer. Math. Soc. 28 (2015), no. 1, 183-197, 199-234, 235-278. MR-3264766; MR-3264767; MR-3264768

[14] X. Chen and B. Wang, Space of Ricci flows (II)-Part A: Moduli of singular Calabi-Yau spaces, Forum Math. Sigma 5 (2017), e32, 103 pp. MR-3739253

[15] X. Chen and B. Wang, Space of Ricci flows (II)-Part B: Weak compactness of the flows, J. Differential Geom., to appear.

[16] J. Chu, V. Tosatti, and B. Weinkove, $C^{1,1}$ regularity for degenerate complex Monge-Ampère equations and geodesic rays, Comm. Partial Differential Equations 43 (2018), no. 2, 292-312. MR-3777876

[17] J. Chu and B. Zhou, Optimal regularity of plurisubharmonic envelopes on compact Hermitian manifolds, Sci. China Math. 62 (2019), no. 2, 371-380. MR-3915068

[18] D. Coman and V. Guedj, Quasiplurisubharmonic Green functions, J. Math. Pures Appl. (9) 92 (2009), no. $5,456-475$. MR- 2558420

[19] C. B. Croke, Some isoperimetric inequalities and eigenvalue estimates, Ann. Sci. École Norm. Sup. (4) 13 (1980), no. 4, 419-435. MR-0608287

[20] V. Datar and G. Székelyhidi, Kähler-Einstein metrics along the smooth continuity method, Geom. Funct. Anal. 26 (2016), no. 4, 975-1010. MR-3558304

[21] J.-P. Demailly, Mesures de Monge-Ampère et caractérisation géométrique des variétés algébriques affines, Mém. Soc. Math. France (N.S.), No. 19, 1985. MR-0813252 
[22] J.-P. Demailly, Regularization of closed positive currents and intersection theory, J. Algebraic Geom. 1 (1992), no. 3, 361-409. MR-1158622

[23] S. Dinew, V. Guedj, and A. Zeriahi, Open problems in pluripotential theory, Complex Var. Elliptic Equ. 61 (2016), no. 7, 902-930. MR-3500508

[24] S. K. Donaldson and S. Sun, Gromov-Hausdorff limits of Kähler manifolds and algebraic geometry, Acta Math. 213 (2014), no. 1, 63-106. MR-3261011

[25] R. C. Gunning, Introduction to holomorphic functions of several variables. Vol. I. Function theory, The Wadsworth \& Brooks/Cole Mathematics Series. Wadsworth \& Brooks/Cole Advanced Books \& Software, Pacific Grove, CA, 1990. MR-1052649

[26] C. Li, On the limit behavior of metrics in the continuity method for the Kähler-Einstein problem on a toric Fano manifold, Compos. Math. 148 (2012), no. 6, 1985-2003. MR-2999312

[27] P. Li, On the Sobolev constant and the p-spectrum of a compact Riemannian manifold, Ann. Sci. École Norm. Sup. (4) 13 (1980), no. 4, 451-468. MR-0608289

[28] N. McCleerey, Envelopes with prescribed singularities, preprint 2018. arXiv:1807.05817

[29] A. M. Nadel, Multiplier ideal sheaves and Futaki's invariant. In: Geometric theory of singular phenomena in partial differential equations (Cortona, 1995), pp. 7-16, Sympos. Math., XXXVIII, Cambridge Univ. Press, Cambridge, 1998. MR-1702085

[30] D. H. Phong and J. Sturm, On the singularities of the pluricomplex Green's function. In: Advances in analysis: the legacy of Elias M. Stein, pp. 419-435, Princeton Math. Ser., vol. 50, Princeton Univ. Press, Princeton, NJ, 2014. MR-3329859

[31] A. Rashkovskii and R. Sigurdsson, Green functions with singularities along complex spaces, Internat. J. Math. 16 (2005), no. 4, 333-355. MR-2133260

[32] J. Ross and D. W. Nyström, Envelopes of positive metrics with prescribed singularities, Ann. Fac. Sci. Toulouse Math. (6) 26 (2017), no. 3, 687-728. MR-3669969

[33] Y. Shi and X. Zhu, An example of a singular metric arising from the blow-up limit in the continuity approach to Kähler-Einstein metrics, Pacific J. Math. 250 (2011), no. 1, 191-203. MR-2780393

[34] J. Siciak, Wiener's Type Sufficient Conditions in $\mathbb{C}^{N}$, Univ. Iagel. Acta Math. 35 (1997), 47-74. MR1458044

[35] Y.-T. Siu, The existence of Kähler-Einstein metrics on manifolds with positive anticanonical line bundle and a suitable finite symmetry group, Ann. of Math. (2) 127 (1988), no. 3, 585-627. MR-0942521

[36] G. Székelyhidi, Greatest lower bounds on the Ricci curvature of Fano manifolds, Compos. Math. 147 (2011), no. 1, 319-331. MR-2771134

[37] G. Székelyhidi, The partial $C^{0}$-estimate along the continuity method, J. Amer. Math. Soc. 29 (2016), no. 2, 537-560. MR-3454382

[38] G. Tian, On Kähler-Einstein metrics on certain Kähler manifolds with $C_{1}(M)>0$, Invent. Math. 89 (1987), no. 2, 225-246. MR-0894378

[39] G. Tian, On Calabi's conjecture for complex surfaces with positive first Chern class, Invent. Math. 101 (1990), no. 1, 101-172. MR-1055713 
[40] G. Tian, K-stability and Kähler-Einstein metrics, Comm. Pure Appl. Math. 68 (2015), no. 7, 1085-1156. MR-3352459 Corrigendum: Ibid. 68 (2015), no. 11, 2082-2083. MR-3403760

[41] G. Tian and S.-T. Yau, Kähler-Einstein metrics on complex surfaces with $C_{1}>0$, Comm. Math. Phys. 112 (1987), no. 1, 175-203. MR-0904143

[42] V. Tosatti, Adiabatic limits of Ricci-flat Kähler metrics, J. Differential Geom. 84 (2010), no. 2, 427-453. MR-2652468

[43] V. Tosatti, Kähler-Einstein metrics on Fano surfaces, Expo. Math. 30 (2012), no. 1, 11-31. MR-2899654

[44] V. Tosatti, Blowup behavior of the Kähler-Ricci flow on Fano manifolds, Univ. Iagel. Acta Math. 50 (2012), 117-126. MR-3235007

[45] V. Tosatti, Regularity of envelopes in Kähler classes, Math. Res. Lett. 25 (2018), no. 1, 281-289. MR3818623

[46] S.-T. Yau, On the Ricci curvature of a compact Kähler manifold and the complex Monge-Ampère equation. I, Comm. Pure Appl. Math. 31 (1978), no. 3, 339-411. MR-0480350 